\title{
TRPM8 genetic variant is associated with chronic migraine and allodynia
}

\author{
Yu-Hsiang Ling ${ }^{1,2}$, Shih-Pin Chen ${ }^{1,2,3,4,5}$, Cathy Shen-Jang Fann ${ }^{6}$, Shuu-Jiun Wang ${ }^{1,2,4}$ and Yen-Feng Wang ${ }^{1,2,4^{*}}$
}

\begin{abstract}
Background: Many single nucleotide polymorphisms (SNPs) have been reported to be associated with migraine susceptibility. However, evidences for their associations with migraine endophenotypes or subtypes are scarce. We aimed to investigate the associations of pre-identified migraine susceptibility loci in Taiwanese with migraine endophenotypes or subtypes, including chronic migraine and allodynia.

Methods: The associations of six SNPs identified from our previous study, including TRPM8 rs10166942, LRP1 rs1172113, DLG2 rs655484, GFRA1 rs3781545, UPP2 rs7565931, and GPR39 rs10803531, and migraine endophenotypes, including chronic migraine and allodynia were tested. Significant associations in the discovery cohort were validated in the replication cohort. The adjusted odds ratios (aOR) were calculated after controlling for confounders.

Results: In total, 1904 patients (mean age $37.5 \pm 12.2$ years old, female ratio: 77.7\%) including 1077 in the discovery cohort and 827 in the replication cohort were recruited. Of them, 584 (30.7\%) had chronic migraine. Of the 6 investigated SNPS, TRPM8 rs10166942 T allele-carrying patients were more likely to have chronic migraine than non-T allele carriers in both discovery and replication cohorts and combined samples (33.7\% vs. $25.8 \%, p=0.004, \mathrm{aOR}=1.62)$. In addition, T allele carriers reported more allodynic symptoms than non-T allele carriers ( $3.5 \pm 3.7$ vs. $2.6 \pm 2.8, p<$ 0.001). However, allodynia severity did not differ between episodic and chronic migraine patients. No further correlations between genetic variants and endophenotypes were noted for the other SNPS.
\end{abstract}

Conclusions: TRPM8 may contribute to the pathogenesis of chronic migraine. However, our study did not support allodynia as a link between them. The underlying mechanisms deserve further investigations.

Keywords: Single nucleotide polymorphism, rs10166942, Pain sensitisation

\section{Introduction}

Migraine, which is characterised by recurrent pulsatile headaches associated with nausea, vomiting, photophobia, and phonophobia, is a common yet disabling disease [1] that can be clinically diagnosed using the proposed criteria in the International Classification of Headache Disorders (ICHD) [2]. Migraine is considered as a complex genetic disorder. Studies of twins and familial aggregation analyses indicate a strong genetic component in migraine, showing a heritability of $0.34-0.81$ [3-8] that can be attributed to polygenes with a modest effect $[9,10]$. Several single nucleotide polymorphisms (SNPs) associated with migraine

\footnotetext{
*Correspondence: yfwang851106@gmail.com

'Department of Neurology, Neurological Institute, Taipei Veterans General Hospital, Taipei, Taiwan

${ }^{2}$ Faculty of Medicine, School of Medicine, National Yang-Ming University,

Taipei, Taiwan

Full list of author information is available at the end of the article
}

susceptibility were recently identified by genome-wide association studies (GWAS) [11]. In our study among the Han Chinese population in Taiwan, several novel variants were identified to be associated with migraine in a two-stage GWAS [12], including rs655484 in disks large homolog 2 (DLG2) and rs3781545 in GDNF family receptor alpha-1 (GFRA1), rs10803531 in G protein-coupled receptor 39 (GPR39), and rs7565931 in uridine phosphorylase 2 (UPP2). Furthermore, the association between migraine and rs10166942 in transient receptor potential melastatin 8 (TRPM8) as well as rs1172113 in low density lipoprotein receptor-related protein 1 (LRP1), the two most replicated SNPs in Caucasians, were also reproduced in our study cohort. The risk allele frequencies are listed in Additional file 1: Table S1.

Endophenotypes are clinical symptoms that differentiate patients into subdivisions with underlying genetic 
pathogenesis. Several endophenotypes have been proposed in migraine, including prodromal symptoms such as yawning, aura, or accompanying symptoms like nausea, vomiting and pulsating. Unilateral autonomic symptoms during attacks are also raised as endophenotypes of migraine [13]. The association of some endophenotypes and migraine implicit genotypes have been studied and reported [14, 15]. Among all migraine endophenotypes or subtypes, chronic migraine is the one with critical clinical significance because it locates at the end of more severe disease-related disability with a poorer quality of life [16]. The prevalence of chronic migraine ranges from $1.0 \%$ to $1.7 \%$ in Asian populations $[17,18]$. However, no susceptible gene has been identified to be associated with chronic migraine to date. On the other hand, allodynia is another widely-studied endophenotype of migraine. Defined as the pain triggered by a normally innocuous stimulation, cutaneous allodynia is very common in migraine patients [19-21], especially in patients with chronic migraine [22]. The presence of allodynia often represents the peripheral, central, and disinhibitory sensitisation of pain pathways in affected patients [23].

In the present study, we aimed to investigate the association between migraine endophenotypes, especially for chronic migraine and allodynia, and known susceptible genes of migraine in Taiwan. Other investigated endophenotypes included aura and migrainous features. The candidate genes were chosen based on the findings of our previous migraine GWAS, the only published study in Asians [12]. The current study adopted a two-stage design, including discovery and replication cohorts of patients with migraine. In the replication cohort, we also evaluated the cutaneous allodynia profile in patients with migraine using a 17-item questionnaire, aiming to obtain the evidence for the association between genetic variants and cutaneous allodynia, a clinical marker signaling the sensitisation of trigeminovascular system and a potential predictor of migraine chronification [19].

\section{Materials and methods \\ Ethics}

The study was approved by the Institutional Review Board of the Taipei Veterans General Hospital, Taipei, Taiwan (IRB No. 2011-11-002GA). Written informed consent was obtained from each participant prior to entering the trial. All clinical investigations were conducted according to the principles expressed in the Declaration of Helsinki. All collected information was de-identified before statistical analysis. The corresponding author had full access to all of the data in the study and had final responsibility for the decision to submit the study for publication.

\section{Study participants and data collection}

This was a two-stage study consisting of a discovery cohort and a replication cohort. All patients with migraine were recruited from the headache clinic of Taipei Veterans General Hospital, Taipei, Taiwan. The discovery and replication cohorts were recruited based on the time they entered the study and whether they completed the allodynia assessment. For the discovery and replication cohorts, we prospectively recruited patients from January 2011 to December 2014 and January 2014 to May 2017 respectively. The sample size was reached based on the numbers of unrelated participants during the study period. All participants completed a detailed headache intake form prior to joining the study and then partook in a semi-structured interview conducted by headache specialists; the participants' demographics, headache characteristics and endophenotypes, medical history, and mental condition assessment were obtained during the interview. The diagnoses of migraine and chronic migraine were made based on the ICHD-3 criteria [2]. The level of anxiety and depression was measured by the Hospital Anxiety and Depression Scale (HADS); the quality of sleep was measured by the Pittsburgh Sleep Quality Index (PSQI).

\section{Genotyping}

Based on the finding of our prior work [12], all participants in discovery cohort were genotyped for six SNPs known to be associated with migraine in Taiwanese, including rs10166942 in TRPM8, rs1172113 in LRP1, rs7565931 in UPP2, rs10803531 in GPR39, rs655484 in $D L G 2$, and rs3781545 in GFRA1. In the replication cohort, every participant was genotyped for rs10166942 in TRPM8-the only significant one identified in the discovery cohort. The genotyping was conducted using the Sequenom MassARRAY iPLEX platform (Sequenom Inc., San Diego, CA, USA) in collaboration with the National Centre for Genomics Medicine, Academia Sinica, Taiwan. Of note, not all the genetic variants identified from migraine GWAS in Western populations were genotyped in this study because our previous study has demonstrated that except for the SNPs in TRPM8 and $L R P 1$, none of the other variants could be replicated in our population with this sample size [12].

\section{Allodynia assessment}

We assessed the allodynia profile of all participants in the replication cohort. Using the 17-item allodynia assessment questionnaire specific for migraine patients, the participants were asked to recall any allodynic symptoms they had during a migraine attack [24]. The items were as followed: (1) combing hair; (2) pulling hair backward (example: ponytail); (3) shaving face; (4) wearing glasses; (5) wearing contact lens; (6) wearing earrings; (7) wearing necklaces; (8) wearing anything on head or neck; (9) wearing anything on arms or wrists; (10) wearing a finger ring; (11) wearing tight clothes; (12) wearing a watch; (13) being covered by a heavy blanket; (14) pouring water onto the 
face; (15) resting the face ipsilateral to the headache side during a headache attack; (16) being exposed to heat (e.g., cooking); and (17) being exposed to cold (e.g., breathing through the nose on a cold day). The total allodynia score was calculated to represent the severity of allodynia by summing up all allodynia symptoms for one point each.

\section{Statistical analysis}

The descriptive statistics were presented as the mean \pm standard deviation (SD), or as percentages. Unanswered queries in the questionnaire were assigned as missing values and excluded from subsequent analyses. Categorical variables were compared between groups using the chi-square test. Continuous variables were compared between groups using Student $t$-test or one-way analysis of variance (ANOVA). Based on the genotyping results, the participants were classified into two groups: (1) risk allele carriers, and (2) non-risk allele carriers. The odds ratios were calculated for risk allele carriers versus the non-carriers. The associations between genotypes and phenotypes were analyzed; significant associations were further calculated using a general linear model and a logistic regression model. The validation of regression model was assessed using mean square error (MSE) to evaluate the fitness of our model. All statistical analyses were performed on SPSS version 23 (IBM, Armonk, NY, USA) and SAS version 9.4 (SAS Inc., Cary, NC, USA). Statistical significance was defined as $p<0.05$, or as adjusted for the multi-comparison Bonferroni correction.

\section{Data availability}

The data that support the findings of this study are available from the corresponding author on reasonable request.

\section{Results \\ Participants}

In this two-stage genetic association study, we recruited 1077 and 827 patients with migraine in the discovery and replication cohorts, respectively to investigate the associations between chronic migraine and candidate genes (Fig. 1). Overall, 340 (17.9\%) had aura, and 584 $(30.7 \%)$ were diagnosed with chronic migraine. The demographics are shown in Table 1.

\section{Correlation of genotypes and endophenotypes of migraine}

We genotyped all six susceptible variants in all participants and explored their possible associations with endophenotypes of migraine. Among the six investigated SNPs, we found that only TRPM8 variant rs10166942 was associated with chronic migraine. Therefore, this positive association was further investigated by examining all potential confounders. We did not find the association between the rest of genotypes and endophenotypes, including aura, chronic migraine as well as migrainous features (results shown in Additional file 1: Table S2).

\section{Correlations of rs10166942 with chronic migraine}

The proportions of chronic migraine in TRPM8 rs101 $66942 \mathrm{~T}$ allele-carrying patients were significantly higher than non-carriers in either discovery or replication cohorts as well as combined results $(33.7 \%$ vs. $25.8 \%, p<0.001)$,

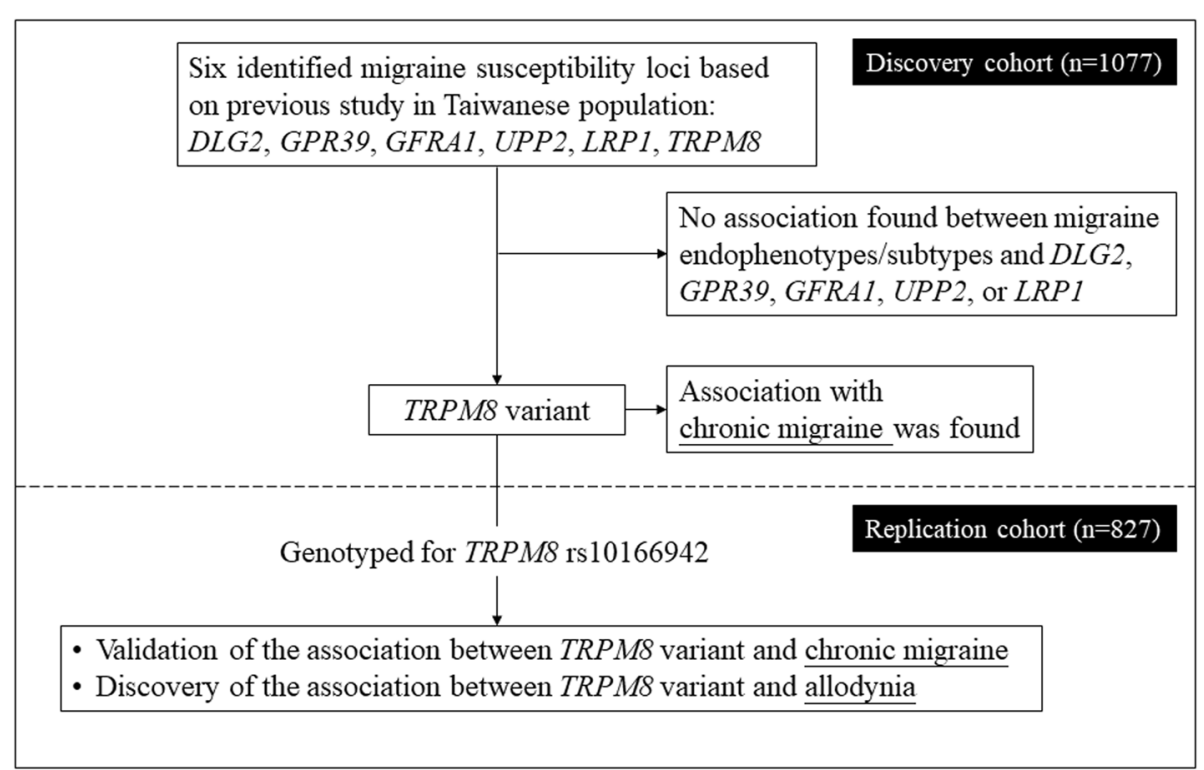

Fig. 1 Study design and main findings of the study 
Table 1 Baseline demographics and clinical data of participants

\begin{tabular}{|c|c|c|c|c|}
\hline & Discovery cohort $(n=1077)$ & Replication cohort $(n=827)$ & $p$ value & $\begin{array}{l}\text { Combined } \\
(n=1904)\end{array}$ \\
\hline Age & $37.9 \pm 12.6$ & $37.0 \pm 11.7$ & 0.116 & $37.5 \pm 12.2$ \\
\hline Female sex & $827(76.8)$ & $653(79.0)$ & 0.259 & $1480(77.7)$ \\
\hline Presence of aura & $180(16.7)$ & $160(19.3)$ & 0.137 & $340(17.9)$ \\
\hline Chronic migraine & $332(30.8)$ & $252(30.5)$ & 0.868 & $584(30.7)$ \\
\hline Disease duration of migraine (years) & $18.4 \pm 11.2$ & $18.1 \pm 11.1$ & 0.591 & $18.2 \pm 11.1$ \\
\hline HADS- anxiety score & $7.6 \pm 4.0$ & $7.4 \pm 4.1$ & 0.176 & $7.5 \pm 4.0$ \\
\hline HADS- depression score & $5.4 \pm 3.7$ & $5.1 \pm 3.7$ & 0.087 & $5.2 \pm 3.7$ \\
\hline PSQI- sleep score & $8.8 \pm 4.0$ & $9.0 \pm 4.0$ & 0.235 & $8.9 \pm 4.0$ \\
\hline
\end{tabular}

Data were presented as $n(\%)$ or mean \pm SD; N: number of participants; HADS: hospital anxiety and depression scale; PSQI: Pittsburgh sleep quality index

and the results remained significant after Bonferroni corrections (Table 2).

The TRPM8 rs10166942 T allele was still independently associated with chronic migraine (adjusted odds ratio $=1.62, p=0.004$ ) after controlling for age, gender, education, body mass index, depression and anxiety using a multivariable logistic regression (Table 3) in both discovery and replication cohorts.

\section{Allodynia assessment}

Primarily, the total allodynia score was higher in the migraine participants carrying the $\mathrm{T}$ allele than in those without ( $3.5 \pm 3.7$ vs. $2.6 \pm 2.8, p<0.001$ ) (Table 2). After controlling for age, gender, chronic migraine, disease duration of migraine, and level of anxiety and depression (HADS anxiety and depression score), rs10166942 T allele carriers were still associated with total allodynia scores versus non-T allele carriers $(p=0.001$, Table 4$)$. Of note, we did not find significant difference in the severity of allodynia between participants with and without chronic migraine (3.2 \pm 3.2 vs. $3.1 \pm 3.4, p=0.623)$. In addition, we found that $\mathrm{T}$ allele carriers were more susceptible to allodynia that was provoked by wearing eyeglasses, wearing contact lenses, wearing earrings, wearing necklaces, wearing anything on the wrist or forearm, wearing a ring, pouring water on the face, exposure to heat, and breathing through nose on cold days than homozygous $\mathrm{C}$ allele carriers (Additional file 1: Table S3).

The distribution of headache frequencies and allodynia severity in migraineurs with or without $\mathrm{T}$ allele rs10166942 is illustrated in Fig. 2, showing that patients with $\mathrm{T}$ allele in rs10166942 tend to have a higher headache frequency or more severe allodynia than those without.

\section{Discussion}

To the best of our knowledge, this study first demonstrates that the TRPM8 rs10166942 T allele-also known

Table 2 Migraine endophenotypes and TRPM8 genotyping in participants

\begin{tabular}{|c|c|c|c|c|c|c|c|}
\hline & \multicolumn{3}{|c|}{ Discovery cohort $(N=1077)$} & \multicolumn{3}{|c|}{ Replication cohort $(N=827)$} & \multirow{2}{*}{$\begin{array}{l}\text { Combined } \\
p \text { value }\end{array}$} \\
\hline & $\begin{array}{l}\mathrm{T} / \mathrm{T} \text { or T/C } \\
n=681(63.2 \%)\end{array}$ & $\begin{array}{l}C / C \\
n=396(36.8 \%)\end{array}$ & $p$ value & $\begin{array}{l}\mathrm{T} / \mathrm{T} \text { or } \mathrm{T} / \mathrm{C} \\
n=495 \\
(59.8 \%)\end{array}$ & $\begin{array}{l}C / C \\
n=332(40.2 \%)\end{array}$ & $p$ value & \\
\hline Age & $38.2 \pm 12.8$ & $37.3 \pm 12.2$ & 0.219 & $37.1 \pm 11.9$ & $36.9 \pm 11.4$ & 0.861 & 0.267 \\
\hline Gender- female & $515(75.6)$ & $312(78.8)$ & 0.236 & $398(80.4)$ & $255(76.8)$ & 0.214 & 0.899 \\
\hline Presence of aura & $102(15.0)$ & $78(19.7)$ & 0.045 & $91(18.4)$ & $69(20.8)$ & 0.392 & 0.036 \\
\hline Chronic migraine & $228(33.5)$ & $104(26.3)$ & 0.013 & $168(33.9)$ & $84(25.3)$ & 0.008 & $<0.001^{*}$ \\
\hline Unilaterality & $513(75.3)$ & $303(76.5)$ & 0.662 & $406(82.0)$ & $273(82.2)$ & 0.939 & 0.676 \\
\hline Pulsatility & $500(74.5)$ & $291(74.4)$ & 0.974 & $386(78.0)$ & $256(77.1)$ & 0.768 & 0.923 \\
\hline Aggravation by or avoidance of physical activity & $599(89.4)$ & $346(89.2)$ & 0.908 & $441(89.1)$ & $276(83.1)$ & 0.013 & 0.057 \\
\hline Nausea & $589(87.5)$ & $350(89.3)$ & 0.389 & $445(89.9)$ & $297(89.5)$ & 0.838 & 0.823 \\
\hline Vomiting & $305(45.3)$ & $177(45.2)$ & 0.958 & $263(53.1)$ & $165(49.7)$ & 0.333 & 0.826 \\
\hline Photophobia & $342(50.8)$ & $188(48.1)$ & 0.390 & $246(49.7)$ & $158(47.6)$ & 0.552 & 0.512 \\
\hline Phonophobia & $507(75.3)$ & $294(75.0)$ & 0.903 & $359(72.5)$ & $234(70.5)$ & 0.523 & 0.839 \\
\hline Allodynia severity & - & - & - & $3.5 \pm 3.7$ & $2.6 \pm 2.8$ & $<0.001^{*}$ & - \\
\hline
\end{tabular}

Data were presented as $n$ (\%) or mean \pm SD. T/T: TT homozygous group; T/C: TC or CT heterozygous group; C/C: CC homozygous group; HADS: hospital anxiety and depression scale; * significant after Bonferroni correction $(p<0.05 / 12=0.0042)$; allodynia severity was presented with the total allodynia scores 
Table 3 Demonstration of the associations between chronic migraine and rs10166942 genotypes by logistic regression models

\begin{tabular}{|c|c|c|c|c|}
\hline & \multicolumn{2}{|c|}{ Univariate } & \multicolumn{2}{|c|}{ Multivariable $^{a}$} \\
\hline & OR & $p$ value & OR & $p$ value \\
\hline \multicolumn{5}{|l|}{ Discovery cohort } \\
\hline Age & 1.03 & $<0.001^{* *}$ & 1.02 & $0.001^{* * *}$ \\
\hline Sex $($ female $=1 /$ male $=0)$ & 1.29 & 0.116 & - & - \\
\hline Education (years) & 0.92 & $<0.001^{* *}$ & 0.83 & $<0.001^{* *}$ \\
\hline Level of anxiety & 1.06 & $<0.001^{* *}$ & - & - \\
\hline Level of depression & 1.09 & $<0.001^{* *}$ & - & - \\
\hline rs $10166942($ T carriers $=1)$ & 1.41 & $0.014^{*}$ & 1.40 & $0.020^{*}$ \\
\hline \multicolumn{5}{|l|}{ Replication cohort } \\
\hline Age & 1.04 & $<0.001^{* *}$ & 1.02 & $0.006^{* *}$ \\
\hline Sex $($ female $=1 /$ male $=0)$ & 1.28 & 0.194 & - & - \\
\hline Education (years) & 0.84 & $<0.001^{* *}$ & 0.70 & $<0.001^{* *}$ \\
\hline BMI & 1.01 & 0.517 & - & - \\
\hline Level of anxiety & 1.05 & $0.008^{* *}$ & - & - \\
\hline Level of depression & 1.11 & $<0.001^{* *}$ & 1.10 & $0.001^{* *}$ \\
\hline rs $10166942($ T carriers $=1)$ & 1.52 & $0.008^{* *}$ & 1.56 & $0.008^{* *}$ \\
\hline \multicolumn{5}{|l|}{ Combined } \\
\hline Age & 1.03 & $<0.001^{* *}$ & 1.02 & $0.019^{*}$ \\
\hline Sex $($ female $=1 /$ male $=0)$ & 1.29 & $0.042^{*}$ & - & - \\
\hline Education (years) & 0.89 & $<0.001^{* *}$ & 0.71 & $<0.001^{* *}$ \\
\hline BMI & 1.01 & 0.529 & - & - \\
\hline Level of anxiety & 1.06 & $<0.001^{* *}$ & - & - \\
\hline Level of depression & 1.09 & $<0.001^{* *}$ & 1.11 & $<0.001^{* *}$ \\
\hline rs10166942 (T carriers =1) & 1.46 & $0.001^{* *}$ & 1.62 & $0.004^{* *}$ \\
\hline
\end{tabular}

a controlled by age, sex and anxious level. $O R$ odds ratio; $B M I$ body mass index; level of anxiety: presented by HADS anxiety score; level of depression: presented by HADS depression score. ${ }^{*}$ indicated $p<0.05 ;{ }^{* *}$ indicated $p<0.01$. Of note, BMI data were not available in discovery cohort

Table 4 Demonstration of the associations between allodynia severity and rs10166942 genotypes by general linear models

\begin{tabular}{|c|c|c|c|c|}
\hline & \multicolumn{2}{|c|}{ Univariate } & \multicolumn{2}{|c|}{ Multivariable $^{a}$} \\
\hline & B & $p$ value & B & $p$ value \\
\hline Age & 0.008 & 0.443 & -0.035 & $0.019^{*}$ \\
\hline Sex $($ female $=1 /$ male $=0)$ & 1.230 & $<0.001^{* *}$ & 1.079 & $<0.001^{* *}$ \\
\hline Chronic migraine & 0.126 & 0.623 & - & - \\
\hline Disease duration of migraine (years) & 0.032 & $0.003^{* *}$ & 0.054 & $0.001^{* *}$ \\
\hline Level of anxiety & 0.135 & $<0.001^{* *}$ & 0.103 & $0.007^{*}$ \\
\hline Level of depression & 0.108 & $0.001^{* *}$ & - & - \\
\hline rs10166942 (T carriers =1) & 0.888 & $0.001^{* *}$ & 0.776 & $0.001^{* *}$ \\
\hline
\end{tabular}

${ }^{a}$ controlled by age, sex and depressive level. $B$ B coefficient; level of anxiety was presented by HADS anxiety score; level of depression was presented by HADS depression score. ${ }^{*} p<0.05 ;{ }^{* *} p<0.01$ as the risk allele of migraine-makes migraine patients more susceptible to migraine chronification. This finding was confirmed by a two-stage validation with two independent cohorts. In addition, our study also demonstrates the association between the TRPM8 rs10166942 $\mathrm{T}$ allele and allodynia severity. However, our study did not find a difference in allodynia severity between patients with episodic and chronic migraine.

The pathophysiology of chronic migraine is an urgent issue to be solved. Prior attempts to search for the relationship between genetic polymorphism, including another TRPM8 variant rs17862920, and chronic migraine have failed [25]. However, our study demonstrated an association between the rs10166942 $\mathrm{T}$ allele and chronic migraine with an adjusted odds ratio of 1.62 , even after controlling for other known confounding factors. This finding deserves verification by following up with patients with episodic migraine in a longitudinal design. Several factors are considered as being involved in the process of migraine chronification, including older age, female gender, lower educational status, obesity, depression, stress and poor response to acute treatment [26]. In line with prior studies, our data showed that older age, lower education and higher depressive levels were risk factors for chronic migraine.

In animal models of craniofacial pain, two studies have demonstrated the association between TRPM8 and allodynia $[27,28]$. However, the association between the TRPM8 genetic variant and allodynia has never been reported in human studies with migraine. Our study can be considered as a translational research, i.e. from animal models to human patients. Our data showed that severity of allodynia in migraineurs was positively associated with being female, longer disease duration of migraine, and psychiatric comorbidities, including depression and anxiety. These findings were also consistent with previous studies [29, $30]$, indicating that our cohort is a representative sample for migraine.

Of note, unlike a previous study [30], our data did not show associations between chronic migraine and the severity of allodynia. In fact, the results of the associations between allodynia and chronic migraine remained undetermined. One paediatric study also did not demonstrate an association between allodynia and chronic migraine [31]. In addition, one study showed that the threshold of pressure pain as measured by quantitative sensory tests did not differ between women with episodic and chronic migraine [32]. Recent results from MAST (Migraine in America Symptoms and Treatment) study showed that increased headache frequency was associated with allodynia; however, the effect was attenuated after controlling for sociodemographic profiles [33]. Regardless of the discrepancy, our results showed that allodynia was positively associated with the disease 


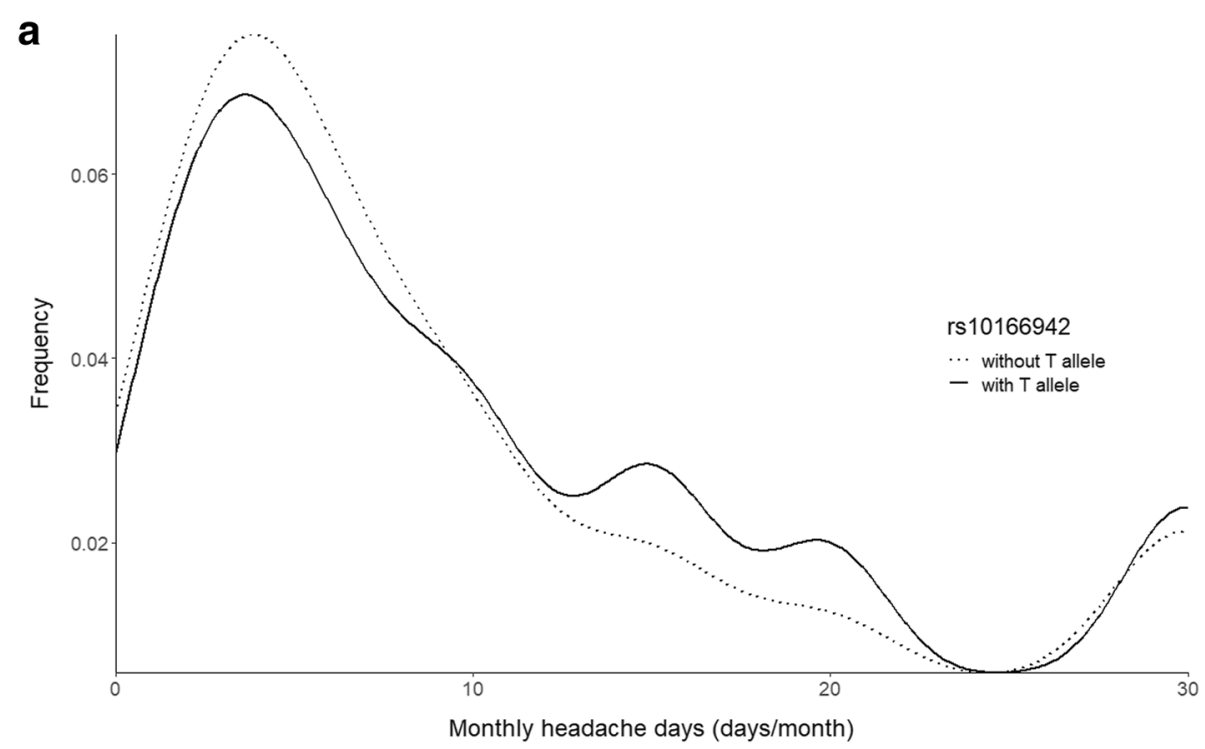

b

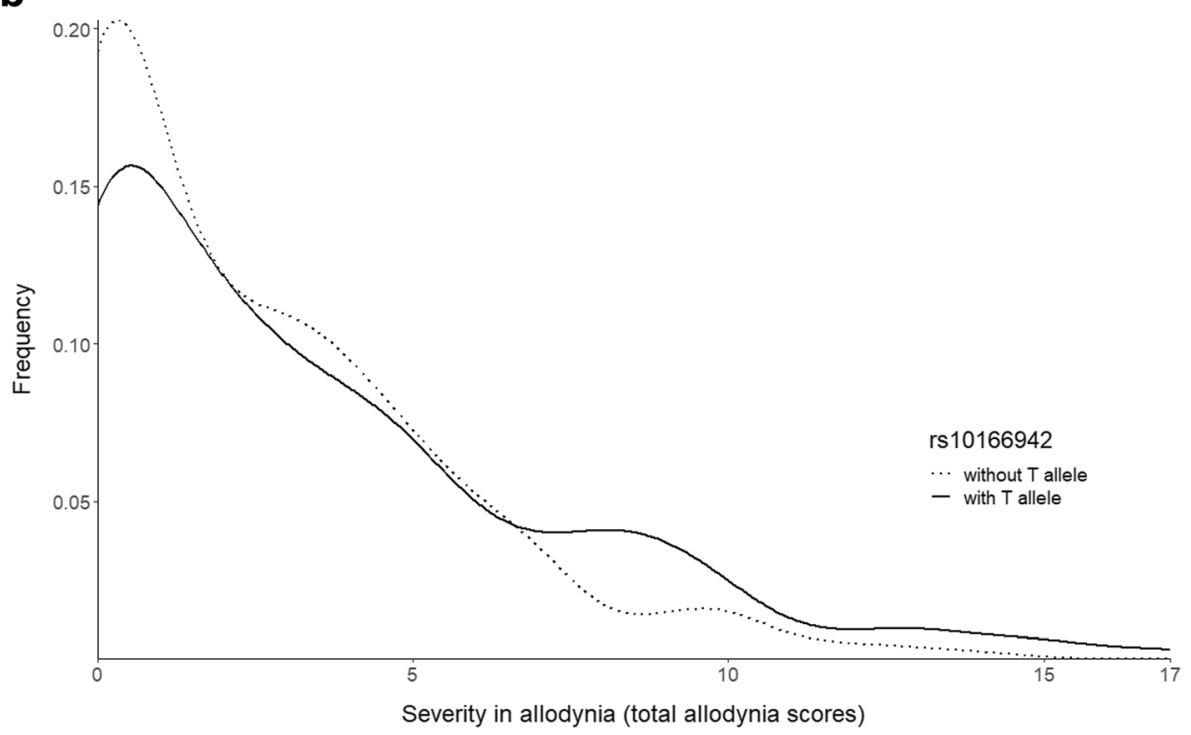

Fig. 2 a The distribution of headache frequencies in migraineurs with or without T allele in rs 10166942. b The distribution of allodynia severity in migraineurs with or without T allele in rs10166942

duration of migraine, which is in line with a previous study [21].

Encoded by gene TRPM8, the TRPM8 receptor is a non-selective cation channel that serves as the primary sensor of cold and cold-induced pain in mammals. The receptor can be activated by cold ranging from 8 to $28^{\circ} \mathrm{C}$ and cooling agents, including methanol and icilin [34]. The $\mathrm{T}$ allele in the TRPM 8 variant rs10166942 is a risk allele for migraine, and our results further indicate that it is also a risk allele to allodynia in migraineurs. The association between the TRPM8 variant rs10166942 and migraine was initially found in Western populations and replicated later in Asians [12]; here, the $\mathrm{T}$ allele was the risk allele for migraine across all studies [11]. To date, evidence in level of molecular mechanism that determines the functional effect of rs10166942 is lacking. Based on computational predictions [35], rs10166942 is located at the regulatory region of TRPM8, the functional effect of it might be alterations in the transcriptional regulation of TRPM8 and thus the affecting the phenotypes of patients. Another possibility is that rs10166942 has a strong linkage disequilibrium with the true causal variant, which remains to be identified. A recent study [36] demonstrated that the frequency of $\mathrm{T}$ allele in rs10166942 is positively corelated to the latitude and climate changes, suggesting that $\mathrm{T}$ allelecarrying genetic variant TRPM8 played a role in adaption to cold temperatures. 
We speculate that $\mathrm{T}$ allele of rs10166942 increases the expression level of TRPM8, sensitizes humans' cold sensation, helping them survive from the extreme changes in temperature in high latitude regions. On the other hand, $\mathrm{T}$ allele in rs10166942 may be associated with the functional changes of TRPM8 that contribute to the hypersensitivity to cold sensation and temperature changes. Pathways that transmit signals of cold sensation, including trigeminothalamic tracts, are repeatedly stimulated by cold and temperature changes, and eventually become sensitized in such individuals. The central sensitization of trigeminothalamic system may lead to worsening of cutaneous allodynia, especially to temperature-related stimulations like our investigated cohort reported, and to migraine progression and chronification. Of course, to explore the hypothesis, the exact impact of genotype rs10166942 on TRPM8 expression needs to be elucidated.

The association between TRPM8 and allodynia has been investigated in animal models, but the results are controversial. Kayama et al. revealed that the activation of TRPM8 reversed the heat allodynia in a mouse meningeal inflammation model, indicating that the activation of TRPM8 is protective against allodynia in migraine [28]. The same study also introduced a cell culture model with coexpression of TRPM8 and transient receptor potential cation channel subfamily V member 1 (TRPV1), showing that the activation of TRPV1, which would lead to heat and mechanical allodynia [37], was attenuated by the activation of TRPM8. The work of Kayama et al. suggests that the pathogenesis of allodynia involves multiple nociceptors, which could partially explain how the genetic variant of the cold receptor TRPM8 could be associated with both thermal and mechanical allodynia. Another study showed that the activation of meningeal TRPM8 receptors by the TRPM8 agonist icilin was associated with facial and hind paw mechanical allodynia in rats [27]. The discrepancy in results might be derived from different model systems (topical ilicin in rats vs. inflammatory soup in mice) with different readouts (mechanical vs. thermal allodynia).

Our study has limitations. First, this was a crosssectional study. Therefore, the causal relationship of migraine chronification and TRPM8 variant could not be ascertained. Based on our findings, a longitudinal study is warranted to elucidate the association between the TRPM8 genetic variant rs10166942 and the evolution of migraine. Second, recall bias of allodynia could have been introduced because not all participants were interviewed during headache attacks. Third, the current study was a single hospital-based study despite validation with another independent cohort; therefore, the results might not be generalisable to other migraine populations. Last, the sample size of the present study was limited, and only 6 migraine susceptible SNPs were tested considering a priori evidences (as indicated in the Methods) and the limitation of resources. However, the 6 tested SNPs were proven to be significant in the studied population, which makes our finding indicative, especially in Asian population. Indeed, further investigations of the association between TRPM8 variant and chronic migraine in different populations are warranted.

\section{Conclusions}

Our study shows that the TRPM8 variant rs10166942 is associated with chronic migraine and allodynia in patients with migraine. Further investigation regarding the role of TRPM8 in allodynia pathogenesis and migraine chronification may provide a novel treatment strategy.

\section{Supplementary information}

Supplementary information accompanies this paper at https://doi.org/10 1186/s10194-019-1064-2.

Additional file 1: Table S1. The risk allele frequencies of investigated SNPs. Table S2a. Migraine endophenotypes and LRP1 rs1172113 genotyping Table S2b. Migraine endophenotypes and DLG2 rs655484 genotyping Table S2c. Migraine endophenotypes and GFRA1 rs3781545 genotyping Table S2d. Migraine endophenotypes and UPP2 rs7565931 genotyping. Table S2e. Migraine endophenotypes and GPR39 rs10803531 genotyping. Table S3. Allodynia symptoms during migraine attacks

\section{Abbreviations}

ANOVA: Analysis of variance; aOR: Adjusted odds ratio; DLG2: Disks large homolog 2; GFRA1: GDNF family receptor alpha-1; GPR39: G protein-coupled receptor 39; GWAS: Genome-wide association study; HADS: Hospital Anxiety and Depression Scale; ICHD: International classification of headache disorder; LRP1: Low density lipoprotein receptor-related protein 1; MAST: Migraine in America Symptoms and Treatment; MSE: Mean square error; PSQI: Pittsburgh sleep quality index; SD: Standard deviation; SNP: Single nucleotide polymorphism; TRPM8: Transient receptor potential melastatin 8; TRPV1: Transient receptor potential cation channel subfamily $\vee$ member 1 ; UPP2: Uridine phosphorylase 2

\section{Acknowledgements}

We wish to thank all individuals in this study for their generous participation. We thank the National Center for Genome Medicine of Taiwan for the technical/bioinformatics/statistics support.

\section{Authors' contribution}

YFW had full access to all of the data in the study and take the responsibility for the integrity of the data and the accuracy of the data analysis. SJW, SPC and YFW were involved the study conception and design. YHL and SPC were responsible for acquisition, analysis, and interpretation of data. YHL and SPC were responsible for manuscript drafting. CSJF provided technical and material support. YHL, YFW, SJW, CSJF and SPC contributed to critical revision of the manuscript for important intellectual content. All authors provided the final approval of the version to be published.

\section{Funding}

The authors disclosed receipt of the following financial support for the research, authorship, and/or publication of this article: This work was supported by the Brain Research Center, National Yang-Ming University from The Featured Areas Research Center Program within the framework of the Higher Education Sprout Project by the Ministry of Education (MOE) in Taiwan (to SJW \&SPC); Ministry of Science and Technology of Taiwan [MOST 108-2321-B-010 -014 -MY2 and 108-2321-B-010 001] (to SJW) \& [MOST-1072314-B-010-021] (to SPC); Ministry of Health and Welfare, Taiwan [MOHW108TDU-B-211-133001] (to SJW); Taipei Veterans General Hospital, Taiwan VGH108-C-105] (to SJW) and [VGH-108-C-066] (to SPC). The funders had no role 
in study design, data collection and analysis, decision to publish, or preparation of the manuscript.

\section{Availability of data and materials}

The data that support the findings of this study are available from the corresponding author on reasonable request.

\section{Ethics approval and consent to participate}

The study was approved by the Institutional Review Board of the Taipei Veterans General Hospital, Taipei, Taiwan (IRB No. 2011-11-002GA). Written informed consent was obtained from each participant prior to entering the trial. All clinical investigations were conducted according to the principles expressed in the Declaration of Helsinki. All collected information was deidentified before statistical analysis.

\section{Consent for publication}

Not applicable.

\section{Competing interests}

The authors declared no potential conflicts of interest with respect to the research, authorship, and/or publication of this article.

\section{Author details}

'Department of Neurology, Neurological Institute, Taipei Veterans General Hospital, Taipei, Taiwan. ${ }^{2}$ Faculty of Medicine, School of Medicine, National Yang-Ming University, Taipei, Taiwan. ${ }^{3}$ Institute of Clinical Medicine, National Yang-Ming University, Taipei, Taiwan. ${ }^{4}$ Brain Research Center, National Yang-Ming University, Taipei, Taiwan. ${ }^{5}$ Department of Medical Research, Taipei Veterans General Hospital, Taipei, Taiwan. ${ }^{6}$ Institute of Biomedical Science, Academia Sinica, Taipei, Taiwan.

Received: 8 October 2019 Accepted: 2 December 2019

Published online: 16 December 2019

\section{References}

1. Vos T, Abajobir AA, Abate KH, Abbafati C, Abbas KM, Abd-Allah F et al (2017) Global, regional, and national incidence, prevalence, and years lived with disability for 328 diseases and injuries for 195 countries, 1990-2016: a systematic analysis for the global burden of disease study 2016. Lancet 390(10100):1211-1259

2. Arnold M (2018) Headache classification Committee of the International Headache Society (IHS) the international classification of headache disorders. Cephalalgia 38(1):1-211

3. Gervil M, Ulrich V, Kyvik KO, Olesen J, Russell MB (1999) Migraine without aura: a population-based twin study. Ann Neurol 46(4):606-611

4. Ulrich V, Gervil M, Kyvik KO, Olesen J, Russell MB (1999) Evidence of a genetic factor in migraine with aura: a population-based Danish twin study. Ann Neurol 45(2):242-246

5. Russell MB, Olesen J (1995) Increased familial risk and evidence of genetic factor in migraine. BMJ 311(7004):541-544

6. Mulder EJ, Van Baal C, Gaist D, Kallela M, Kaprio J, Svensson DA et al (2003) Genetic and environmental influences on migraine: a twin study across six countries. Twin Res 6(5):422-431

7. Stam AH, de Vries B, Janssens AC, Vanmolkot KR, Aulchenko YS, Henneman $P$ et al (2010) Shared genetic factors in migraine and depression: evidence from a genetic isolate. Neurology 74(4):288-294

8. Stewart WF, Staffa J, Lipton RB, Ottman R (1997) Familial risk of migraine: a population-based study. Ann Neurol 41(2):166-172

9. de Vries B, Frants RR, Ferrari MD, van den Maagdenberg AM (2009) Molecular genetics of migraine. Hum Genet 126(1):115-132

10. Tolner EA, Houben T, Terwindt GM, de Vries B, Ferrari MD, van den Maagdenberg AM (2015) From migraine genes to mechanisms. Pain 156(Suppl 1):S64-S74

11. Gormley P, Anttila V, Winsvold BS, Palta P, Esko T, Pers TH et al (2016) Metaanalysis of 375,000 individuals identifies 38 susceptibility loci for migraine. Nat Genet 48(8):856

12. Chen S-P, Fuh J-L, Chung M-Y, Lin Y-C, Liao Y-C, Wang Y-F et al (2018) Genome-wide association study identifies novel susceptibility loci for migraine in Han Chinese resided in Taiwan. Cephalalgia 38(3):466-475

13. Barbanti P, Aurilia C, Dall'Armi V, Egeo G, Fofi L, Bonassi S (2016) The phenotype of migraine with unilateral cranial autonomic symptoms documents increased peripheral and central trigeminal sensitization. A case series of 757 patients. Cephalalgia 36(14):1334-1340
14. Nyholt DR, Morley KI, Ferreira MA, Medland SE, Boomsma DI, Heath AC et al (2005) Genomewide significant linkage to migrainous headache on chromosome 5q21. Am J Hum Genet 77(3):500-512

15. Anttila V, Kallela M, Oswell G, Kaunisto MA, Nyholt DR, Hämäläinen E et al (2006) Trait components provide tools to dissect the genetic susceptibility of migraine. Am J Hum Genet 79(1):85-99

16. Wang S-J, Wang P-J, Fuh J-L, Peng K-P, Ng K (2013) Comparisons of disability, quality of life, and resource use between chronic and episodic migraineurs: a clinic-based study in Taiwan. Cephalalgia 33(3):171-181

17. Lu S, Fuh J, Chen W, Juang K, Wang S (2001) Chronic daily headache in Taipei, Taiwan: prevalence, follow-up and outcome predictors. Cephalalgia 21(10):980-986

18. Wang S-J, Fuh J-L, Lu S-R, Liu C-Y, Hsu L-C, Wang P-N et al (2000) Chronic daily headache in Chinese elderly Prevalence, risk factors, and biannual follow-up. Neurology 54(2):314

19. Bigal M, Ashina S, Burstein R, Reed M, Buse D, Serrano D et al (2008) Prevalence and characteristics of allodynia in headache sufferers a population study. Neurology 70(17):1525-1533

20. Kao C-H, Wang S-J, Tsai C-F, Chen S-P, Wang Y-F, Fuh J-L (2014) Psychiatric comorbidities in allodynic migraineurs. Cephalalgia 34(3):211-218

21. Mathew NT, Kailasam J, Seifert T (2004) Clinical recognition of allodynia in migraine. Neurology 63(5):848-852

22. Burstein $\mathrm{R}$, Collins $B$, Jakubowski M (2004) Defeating migraine pain with triptans: a race against the development of cutaneous allodynia. Ann Neurol 55(1):19-26

23. Burstein R, Jakubowski M, Garcia-Nicas E, Kainz V, Bajwa Z, Hargreaves R et al (2010) Thalamic sensitization transforms localized pain into widespread allodynia. Ann Neurol 68(1):81-91

24. Ashkenazi A, Silberstein S, Jakubowski M, Burstein R (2007) Improved identification of allodynic migraine patients using a questionnaire. Cephalalgia 27(4):325-329

25. Louter M, Fernandez-Morales J, de Vries B, Winsvold B, Anttila V, FernandezCadenas I et al (2015) Candidate-gene association study searching for genetic factors involved in migraine chronification. Cephalalgia 35(6):500-507

26. May A, Schulte LH (2016) Chronic migraine: risk factors, mechanisms and treatment. Nat Rev Neurol 12:455

27. Burgos-Vega CC, Ahn DD-U, Bischoff C, Wang W, Horne D, Wang J et al (2016) Meningeal transient receptor potential channel M8 activation causes cutaneous facial and hindpaw allodynia in a preclinical rodent model of headache. Cephalalgia 36(2):185-193

28. Kayama Y, Shibata M, Takizawa T, Ibata K, Shimizu T, Ebine T et al (2018) Functional interactions between transient receptor potential M8 and transient receptor potential $\mathrm{V} 1$ in the trigeminal system: relevance to migraine pathophysiology. Cephalalgia 38(5):833-845

29. Lipton RB, Bigal ME, Ashina S, Burstein R, Silberstein S, Reed ML et al (2008) Cutaneous allodynia in the migraine population. Ann Neurol 63(2):148-158

30. Louter MA, Bosker JE, van Oosterhout WP, van Zwet EW, Zitman FG, Ferrari MD et al (2013) Cutaneous allodynia as a predictor of migraine chronification. Brain 136(11):3489-3496

31. Eidlitz-Markus T, Shuper A, Gorali O, Zeharia A (2007) Migraine and cephalic cutaneous allodynia in pediatric patients. Headache 47(8):1219-1223

32. Palacios-Ceña M, Lima Florencio L, Natália Ferracini G, Barón J, Guerrero ÁL, Ordás-Bandera C et al (2016) Women with chronic and episodic migraine exhibit similar widespread pressure pain sensitivity. Pain Med 17(11):2127-2133

33. Dodick DW, Reed ML, Fanning KM, Munjal S, Alam A, Buse DC et al (2019) Predictors of allodynia in persons with migraine: results from the migraine in America symptoms and treatment (MAST) study. Cephalalgia 39(7):873-882

34. Clapham DE (2003) TRP channels as cellular sensors. Nature 426(6966):517

35. Henström M, Hadizadeh F, Beyder A, Bonfiglio F, Zheng T, Assadi G et al (2017) TRPM8 polymorphisms associated with increased risk of IBS-C and IBS-M. Gut 66(9):1725-1727

36. Key FM, Abdul-Aziz MA, Mundry R, Peter BM, Sekar A, D'Amato $M$ et al (2018) Human local adaptation of the TRPM8 cold receptor along a latitudinal cline. PLoS Genet 14(5):e1007298

37. Yu L, Yang F, Luo H, Liu F-Y, Han J-S, Xing G-G et al (2008) The role of TRPV1 in different subtypes of dorsal root ganglion neurons in rat chronic inflammatory nociception induced by complete Freund's adjuvant. Mol Pain 4(1):61

\section{Publisher's Note}

Springer Nature remains neutral with regard to jurisdictional claims in published maps and institutional affiliations. 